\title{
Assessment of Neuromuscular Blockade in Children at the Time of Block Reversal and the Removal of the Endotracheal Tube
}

\author{
Camila Machado de Souza, TSA 1, Fernanda Elizabeth Romero, TSA 1, Maria Angela Tardelli, TSA 2
}

\begin{abstract}
Summary: Souza CM, Romero FE, Tardelli MA - Assessment of Neuromuscular Blockade in Children at the Time of Block Reversal and the Removal of the Endotracheal Tube.

Background and objectives: Studies show the importance of monitoring neuromuscular function in preventing the residual block. However, most anesthesiologists in their daily practice base their evaluation of the recovery of neuromuscular function on clinical data. The aim of this study was to assess the degree of neuromuscular blockade in children undergoing general anesthesia at the time of block reversal and the removal of the endotracheal tube.
\end{abstract}

Method: We evaluated children between 3 months and 12 years of age undergoing general anesthesia with the use of atracurium or rocuronium. Monitoring was initiated at the time of reversal of neuromuscular blockade and/or removal of the endotracheal tube. The anesthesiologist was not informed about the $T_{4} / T_{1}$ value; he/she was only alerted when the time was inadequate for the removal of the endotracheal tube. Since the start of the monitoring process, the degree of neuromuscular blockade was registered, as well as the interval of recovery of the $T_{4} / T_{1} \geq 0,9$ ratio, the doses of neostigmine and blocker used, the expired fraction of the inhalational agent, the duration of the anesthesia, and core and peripheral temperatures.

Results: Neuromuscular blockade was reversed in $80 \%$ of the children of the Rocuronium Group and in $64.5 \%$ of the Atracurium Group. The reversal was incorrect in $45.8 \%$ of the Rocuronium Group and in $25 \%$ of the Atracurium Group. The incidence of $T_{4} / T_{1}<0.9$ at the time of the removal of the endotracheal tube was $10 \%$ in both groups.

Conclusions: When deciding to remove the endotracheal tube based on clinical criteria, $10 \%$ of children had $\mathrm{T}_{4} / \mathrm{T}_{1}<0.9$ regardless the blocker received. A considerable number of patients had the neuromuscular blockade incorrectly reversed when the blockade was still too deep or even already recovered.

Keywords: Neuromuscular Blocking Agents; Atracurium; Androstanols, rocuronium; Anesthesia, General; Infant; Child; Child, Preschool.

[Rev Bras Anestesiol 2011;61(2): 145-155] @Elsevier Editora Ltda.

\section{INTRODUCTION}

The residual neuromuscular blockade is one of the major complications related to the use of non-depolarizing neuromuscular blockers (NDNMB) during anesthesia. Among the adverse effects of residual blockade are the risk of regurgitation and aspiration of gastric contents, impaired ventilatory response to hypoxia, upper airway obstruction and decreased forced vital capacity ${ }^{1}$. Several studies highlight the importance of objective monitoring of neuromuscular function, in order to prevent the residual block. However, most anesthesiologists in their daily practice base their evaluation of the recovery of neuromuscular function on clinical data, which are subjective.

The reversal of neuromuscular block prior to removal of the endotracheal tube is a way to prevent the adverse effects of re-

Received from the Division of Anesthesiology, Pain and Intensive Care Medicine, Universidade Federal de São Paulo (UNIFESP), Brazil.

1. Assistant Physician, Division of Anesthesiology, Pain and Intensive Care Medicine, UNIFESP

2. Associate Professor in the Division of Anesthesiology, Pain and Intensive Care Medicine, UNIFESP

Submitted on May 31, 2009.

Approved on December 13, 2010.

Correspondence to:

Dra. Camila Machado de Souza

Disciplina de Anestesiologia, Dor e Medicina Intensiva da UNIFESP

Rua Napoleão de Barros, 715, 5o andar

Vila Clementino

04024-002 - São Paulo, SP, Brazil

E-mail:cmdesouza@uol.com.br sidual block. In the absence of monitoring the degree of neuromuscular blockade, authors recommend to routinely antagonize the blockade due to the unpredictability of its spontaneous recovery ${ }^{2,3}$. However, medications used for this purpose are not without side effects. Additionally, there is the fact that the pharmacological reversal of the blockade is not always effective because it involves a competitive mechanism with the NDNMBs ${ }^{1}$.

The incidence of residual block in adults in the anesthesia recovery room has been well documented but few data are available on the same situation in children, because of the difficulties in objective monitoring in this age group.

The aim of this study was to objectively assess with acceleromyography the degree of neuromuscular blockade in children undergoing general anesthesia at the time of block reversal and the removal of the endotracheal tube.

\section{METHODS}

The study was submitted for approval by the Ethics Committee of the Universidade Federal de São Paulo. We evaluated children aged between 3 months and 12 years who underwent surgical procedures under general anesthesia with fentanyl, propofol, isoflurane, atracurium or rocuronium. Monitoring of neuromuscular function was only initiated at the end of the surgical procedure using the Train-of-Four (TOF) through acceleromyography with ulnar nerve stimulation $(20 \mathrm{~mA})$. The intensity 
of neuromuscular blockade was recorded from the time of the decision to reverse the blockade or the decision to remove the endotracheal tube, when the anesthesiologist did not decide for the reversal of the blockade. Furthermore, the interval for recovery of $T_{4} / T_{1} \geq 0.9$ from that moment has also been recorded, as well as the total dose of NDNMB used during the procedure, the core and peripheral temperatures and the total dose of neostigmine, when used. Situations were characterized as "incorrect reversal" when the use of neostigmine was not necessary $\left(T_{4} / T_{1} \geq 0.9\right)$ or would be inefficient (recovery of neuromuscular blockade with less than two responses to TOF stimulation) ${ }^{4,5}$.

The anesthesiologist was aware of the objectives of the study and had no access to the data obtained by the acceleromyography from monitoring neuromuscular function. All decisions regarding the use of NDNMB (dose, dosing interval and block reversal) were taken by the anesthesiologist responsible for the anesthesia without any interference by the investigator, and were based on clinical criteria according to his/her standard practice. The investigator informed the anesthesiologist only the $T_{4} / T_{1}$ value in case it was less than 0.9 at the moment the decision to remove the tracheal tube was made. In this situation, the patient remained intubated and the inhalational agent, if still in use, was shut down pending the recovery of $T_{4} / T_{1}$ to $\geq 0.9$. During this period, hypnosis was maintained with propofol boluses as needed.

Qualitative variables were described as number (n) and percentage (\%), while quantitative measures were expressed by position: median, first quartile (Q1), third quartile (Q3) and the minimum and maximum values. Comparison of the qualitative variables was done using the Chi-square and Fischer's exact tests. For the quantitative variables the Mann-Whitney test was used. Results showing $p$ value of less than $5 \%$ $(p<0.05)$ were considered statistically significant. All statistical analysis was performed with SPSS 16.0 for Windows.

\section{RESULTS}

The demographics of both groups were similar as shown in Table I.

Table I - Demographic Data According to the Group

\begin{tabular}{|c|c|c|c|}
\hline & $\begin{array}{l}\text { Rocuronium } \\
(n=30)\end{array}$ & $\begin{array}{l}\text { Atracurium } \\
(\mathrm{n}=31)\end{array}$ & $p$ \\
\hline Age (months) & & & 0.648 \\
\hline Median (Q1-Q3) & $72(48-96)$ & $72(48-20)$ & \\
\hline Minimum - maximum & $3-132$ & $3-156$ & \\
\hline Weight $(\mathrm{Kg})$ & & & 0.153 \\
\hline Median (Q1-Q3) & $20(14-29)$ & $23(16-35)$ & \\
\hline Minimum - maximum & $3.5-48$ & $5-50$ & \\
\hline Gender & & & 0.344 \\
\hline Male & $20(66.7 \%)$ & $17(54.8 \%)$ & \\
\hline Female & $10(33.3 \%)$ & $14(45.2 \%)$ & \\
\hline Physical state & & & 0.905 \\
\hline ASA I & $21(70.0 \%)$ & $25(80.6 \%)$ & \\
\hline ASA II & $8(26.7 \%)$ & $4(13.0 \%)$ & \\
\hline ASA III & $1(3.3 \%)$ & $0(0 \%)$ & \\
\hline ASA IV & $0(0 \%)$ & $2(6.4 \%)$ & \\
\hline
\end{tabular}

Neuromuscular blockade was reversed with neostigmine at the end of the procedure in $80 \%(n=24)$ of the children of the Rocuronium Group and in $64.5 \%(n=20)$ of the Atracurium Group ( $p=0.178$ ), as shown in Figure 1.

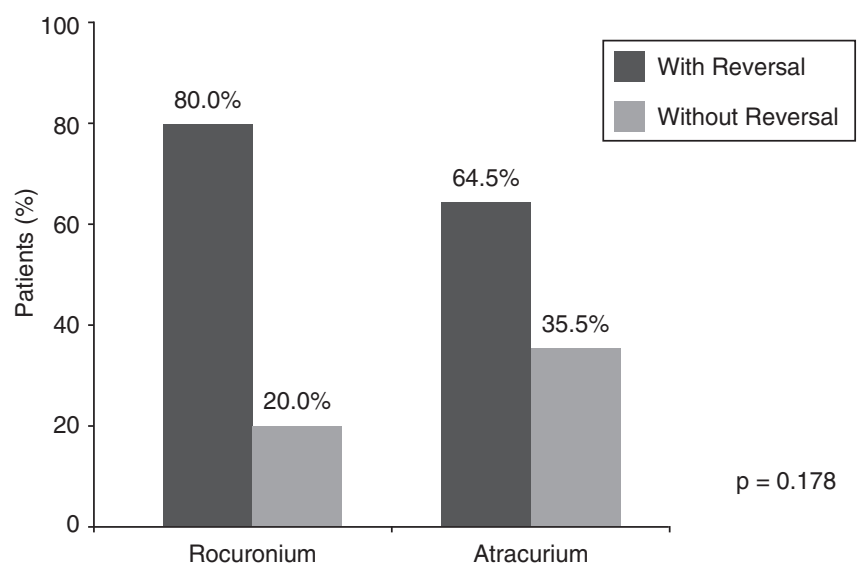

Figure 1 - Distribution of the Decision to Reverse According to the Group.

Among the children who had the blockade reversed ( $n=44)$, the reversal was incorrect in $45.8 \%$ of the Rocuronium Group and in $25 \%$ of the Atracurium Group $(p=0.153)$. The dose of neostigmine was similar in both groups (Table II). The median value of $T_{4} / T_{1}$ at the time of reversal was 0.8 in the Rocuronium Group, and 0.7 in the Atracurium Group. The median time to recovery from block $\left(T_{4} / T_{1} \geq 0.9\right)$ after reversal was 4 minutes in the Rocuronium Group and 5 minutes in the Atracurium Group. There was no difference in the core and peripheral temperatures between the two groups (Table II). Figure 2 shows the distribution of TOF values at the time of reversion in both groups. Among these children, 40 received isoflurane during

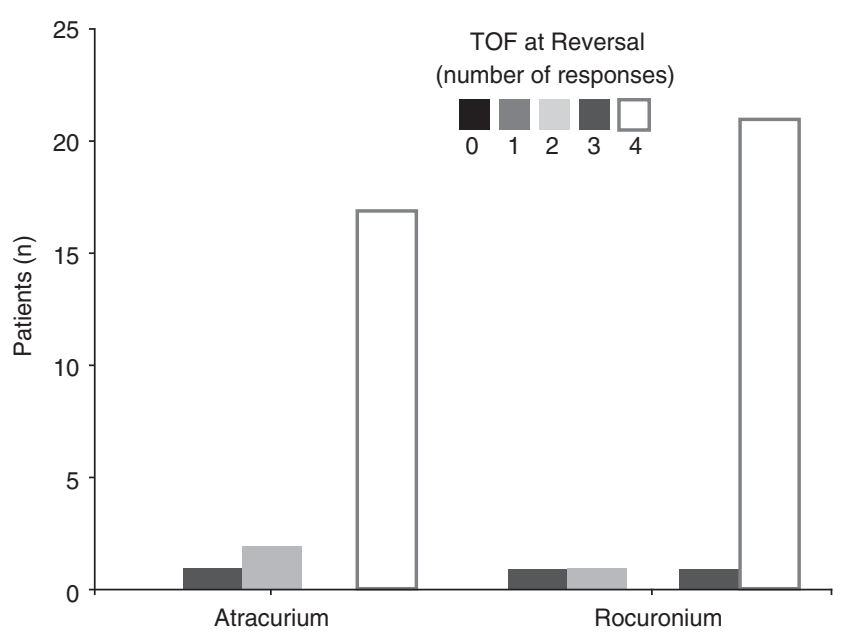

Figure 2 - Distribution of TOF Values in the Reversal According to the Group. 
Table II - Decision to Reverse Neuromuscular Block According to the Group

\begin{tabular}{|c|c|c|c|}
\hline & $\begin{array}{l}\text { Rocuronium } \\
(\mathrm{n}=24)\end{array}$ & $\begin{array}{l}\text { Atracurium } \\
(\mathrm{n}=20)\end{array}$ & $\mathrm{p}$ \\
\hline \multicolumn{4}{|l|}{ Decision to reverse } \\
\hline Correct & $13(54.2 \%)$ & $15(75.0 \%)$ & 0.153 \\
\hline \multicolumn{4}{|l|}{ TOF in incorrect reversal } \\
\hline $\mathrm{T}_{4} / \mathrm{T}_{1} \geq 0.9$ & $10(41.7 \%)$ & $4(20.0 \%)$ & 0.124 \\
\hline TOF $<2$ responses & $1(4.2 \%)$ & $1(5.0 \%)$ & \\
\hline Minimum - maximum & $0.02-0.07$ & $0.02-0.09$ & \\
\hline \multicolumn{4}{|l|}{ Time to $T_{4} / T_{1} \geq 0.9(\mathrm{~min})$} \\
\hline Median (Q1-Q3) & $4(2-10)$ & $5(3-8)$ & 0.983 \\
\hline Minimum - maximum & $2-87$ & $1-18$ & \\
\hline \multicolumn{4}{|l|}{ Core temperature $\left({ }^{\circ} \mathrm{C}\right)$} \\
\hline Minimum - maximum & $29.0-36.0$ & $27.4-36.4$ & \\
\hline \multicolumn{4}{|c|}{ Total dose of NMB $\left(\mathrm{mg} \cdot \mathrm{kg}^{-1}\right)$} \\
\hline Median (Q1-Q3) & $0.79(0.56-1.02)$ & $0.50(0.46-0.52)$ & 0.439 \\
\hline Minimum - maximum & $0.50-2.83$ & $0.43-1.33$ & \\
\hline
\end{tabular}

Q1 - $1^{\text {st }}$ quartile; Q3 $-3^{\text {rd }}$ quartile.

Table III - Patient Data with $\mathrm{T}_{4} / \mathrm{T}_{1}<0.9$ at Extubation

\begin{tabular}{|c|c|c|}
\hline & $\begin{array}{l}\text { Rocuronium } \\
(\mathrm{n}=3)\end{array}$ & $\begin{array}{l}\text { Atracurium } \\
(\mathrm{n}=3)\end{array}$ \\
\hline \multicolumn{3}{|l|}{$\mathrm{T}_{4} / \mathrm{T}_{1}$ at extubation } \\
\hline Median & 0.58 & 0,56 \\
\hline Minimum - maximum & $0.17-0.78$ & $0.37-0.63$ \\
\hline \multicolumn{3}{|l|}{ Time to $T_{4} / T_{1} \geq 0.9(\mathrm{~min})$} \\
\hline Median & 16 & 5 \\
\hline Minimum - maximum & $3-26$ & $3-12$ \\
\hline \multicolumn{3}{|l|}{ Age (months) } \\
\hline Median & 21 & 72 \\
\hline Minimum - maximum & $3-96$ & $60-96$ \\
\hline \multicolumn{3}{|l|}{ Core temperature $\left({ }^{\circ} \mathrm{C}\right)$} \\
\hline Median & 35.0 & 35.8 \\
\hline Minimum - maximum & $35.0-36.8$ & $34.0-37.5$ \\
\hline \multicolumn{3}{|c|}{ Peripheral temperature $\left({ }^{\circ} \mathrm{C}\right)$} \\
\hline Median & 32.1 & 34,1 \\
\hline Minimum-maximum & $30.7-33.1$ & $33.8-34.8$ \\
\hline \multicolumn{3}{|c|}{ Total dose of NMB $\left(\mathrm{mg} \cdot \mathrm{kg}^{-1}\right)$} \\
\hline Median & 1.7 & 0.52 \\
\hline Minimum - maximum & $1-2.83$ & $0.47-0.53$ \\
\hline \multicolumn{3}{|c|}{ Neostigmine dose $\left(\mathrm{mg} \mathrm{kg}^{-1}\right)$} \\
\hline Median & 0.03 & 0.03 \\
\hline Minimum - maximum & $0.07-0.02$ & $0.04-0.02$ \\
\hline \multicolumn{3}{|c|}{ Duration of anesthesia (min) } \\
\hline Median & 185 & 71 \\
\hline Minimum - maximum & $185-412$ & $65-80$ \\
\hline
\end{tabular}

maintenance of anesthesia, and the mean value of halogenate expired fraction was $1.0 \pm 0.24$ in the Rocuronium Group and $0.94 \pm 0.19$ in the Atracurium Group $(p<0.05)$ at the time of reversal. Two children of the Atracurium Group and one of the Rocuronium Group received sevoflurane during maintenance, and values of the halogenate expired fraction at the moment of reversal in these cases were $1.1 \%$ and $1.6 \%$ in the Groups Atracurium and Rocuronium, respectively. One child of the Rocuronium Group received total intravenous anesthesia.

At the time of decision to remove the endotracheal tube, three children of each group had $\mathrm{T}_{4} / \mathrm{T}_{1}<0.9$. The median time to recover the ratio $T_{4} / T_{1} \geq 0.9$ from the moment of the decision to remove the endotracheal tube was 16 minutes in the Rocuronium Group and 5 minutes in the Atracurium Group. These children had received isoflurane during maintenance of anesthesia. However, the values of the halogenate expired fraction at the time of decision to remove the endotracheal tube were not recorded. Other data on these patients are shown in Table III.

Except for one child of the Atracurium Group, all others who presented $T_{4} / T_{1}<0.9$ at the moment of the decision to extubate underwent reversal of neuromuscular blockade with a mean dose of neostigmine of $0.032 \pm 0.02 \mathrm{mg} \cdot \mathrm{kg}^{-1}$. However, the degree of blockade at the time of reversal varied greatly among the children who underwent reversal and that still had $T_{4} / T_{1}<0.9$ at the time of extubation. The values found at the time of reversal were 0 response, 2 responses and 4 
responses in the Rocuronium Group, and 2 responses and $\mathrm{T}_{4} / \mathrm{T}_{1}=0.27$ in the Atracurium Group. The intervals between the reversal and the decision to extubate were 5 minutes and 7 minutes and 61 minutes in the Rocuronium Group, and 3 minutes and 6 minutes in the Atracurium Group, respectively.

\section{DISCUSSION}

This study was designed to evaluate the intensity of neuromuscular blockade at the time of neostigmine administration and/or the removal of the endotracheal tube in children undergoing general anesthesia with use of rocuronium or atracurium without neuromuscular function monitoring.

The residual block at the end of the surgical procedures is a topic which has long been of concern to anesthesiologists ${ }^{6}$. Adequate recovery of neuromuscular function has often been defined as the ability of the patient to keep satisfactory ventilatory function and free airways. Considering the difficulty of using pulmonary function tests in clinical practice that evaluate these goals, such as vital capacity and maximal voluntary ventilation, other tests are used as surrogates. Fatigue of the $\mathrm{T}_{4} / \mathrm{T}_{1}$ ratio was the first suggested for measuring the degree of neuromuscular block and, subsequently, their values have been related to pulmonary function tests ${ }^{6}$.

Studies show that the $T_{4} / T_{1}$ ratio $<0.9$ is associated with worsening of the ventilatory response to hypoxia and of the function of the pharynx and upper esophageal sphincter muscles, predisposing to pulmonary aspiration and respiratory complications ${ }^{1,7}$.

The routine use of neuromuscular function monitoring reduces the incidence of residual block ${ }^{3}$. However, it is important to use monitoring which objectively quantifies the degree of neuromuscular blockade, particularly in its final stages of recovery, because it is very difficult to visually or manually estimate the $T_{4} / T_{1}$ value accurately enough to exclude the residual block ${ }^{8}$. Therefore, in the absence of reliable monitoring, neuromuscular blockade has been routinely reversed with anticholinesterase administration.

The routine reversal of neuromuscular blockade at the end of anesthesia also is no guarantee of restored neuromuscular function at the moment of the endotracheal tube removal. In our study, almost all children who presented with $T_{4} / T_{1}<$ 0.9 at extubation had received a mean dose of $0.03 \mathrm{mg} \cdot \mathrm{kg}^{-1}$ neostigmine for reversal of blockade. The return of neuromuscular function after neostigmine administration is the sum of spontaneous recovery of neuromuscular blockade and its acceleration by anticholinesterase. Consequently, the speed of neuromuscular function restoration after neostigmine administration is affected by the intensity of the blockade at the time of reversal and by the dose of anticholinesterase administered ${ }^{1,8-10}$. When reversal is performed during deep neuromuscular blockade, i.e. in the absence of any detectable muscle response to indirect stimulation, $T_{4} / T_{1}$ ratio recovers the value of 0.4 relatively quickly. However, the recovery of $\mathrm{T}_{4} / \mathrm{T}_{1}>0.7$ does not occur promptly. Therefore, the attempt to reverse deep blockade results in a prolonged window of vulnerability during which the patient may be inadequately re- covered, and the anesthesiologist would be confident that the reversal was successful ${ }^{8}$. Corroborating these data, the study showed that among 40 adults receiving rocuronium or cisatracurium only one in each group had TOF $\geq 0.9$ after 15 minutes of reversal with $0.05 \mathrm{mg} . \mathrm{kg}^{-1}$ neostigmine administered when the blockade had the value of $\mathrm{T} 1=6 \%$. Twenty minutes after the reversal, the $T_{4} / T_{1}$ ratio was 0.83 and 0.79 for those receiving cisatracurium or rocuronium, respectively ${ }^{7}$. This result is not surprising, because cholinesterase inhibitors have shown limited ability to antagonize NDNMBs. If the level of NDNMB in the neuromuscular junction is high enough, the extent of recovery that can be induced is finite.

Considering all the factors that interfere with neuromuscular blockade in its evaluation and reversal, the residual block is still a matter not adequately clarified among anesthesiologists. A study conducted among these professionals in Denmark showed that $91 \%$ underestimated the incidence of residual curarization after intermediate action NDNMB, 45\% knew that TOF $>0.9$ excludes residual curarization, and $13 \%$ never monitored the blockage degree ${ }^{11}$. In Brazil, 92\% of anesthesiologists consider the patient recovered from neuromuscular blockade by clinical signs, 53\% never use monitoring of neuromuscular transmission, and $45 \%$ employ routinely neostigmine ${ }^{12}$.

The evaluation of residual block in children involves certain particularities and limitations. It is difficult to reproduce in children the clinical tests standardized for the adult population, since some degree of collaboration is necessary for its implementation. Acceleromyography is an effective method of monitoring neuromuscular function in children ${ }^{13}$. However, peripheral nerve stimulation can be painful and not tolerated by the awaken patient. Moreover, to ensure accuracy of the measurement recorded by the acceleromyography, patients should not offer resistance to the evoked contraction. It is difficult to meet these criteria in the pediatric population, particularly in post-anesthesia recovery. Additionally, it is important to mention that the physiological changes of pediatric patients account for changes such as fatigue, which is observed in the monitoring of children younger than 12 weeks of age when subjected to tetanic stimulation, even in the absence of NDNMB, and the prolonging of the NDNMB-induced blockade in this age group ${ }^{14,15}$.

In this study, the method used was developed in order to circumvent some of these difficulties when trying to assess residual block in pediatric patients. Although there are no reports on fatigue to the monitoring with TOF in children, we opted to select those with more than 12 weeks of age to avoid interference of physiological changes in the results. The assessment of the $T_{4} / T_{1}$ value in the moment of decision to remove the endotracheal tube was used to circumvent the difficulty of obtaining reliable values of the degree of residual blockade by acceleromyography in children in post-anesthesia recovery. The severity of the blockade at the moment of extubation along with the record of the number of children who presented lower values than 0.9 would reflect the incidence of patients who were at risk of adverse events resulting from the residual block (in case the anesthesiologist was permitted to 
remove the tracheal tube). Thus, recording the time required for neuromuscular function of these patients to recover the value $T_{4} / T_{1} \geq 0.9$ can be inferred as an indirect measure of the duration of residual block in these children. When evaluating the median of these times in Table III, one can speculate that children who had received atracurium, when admitted to the recovery room after anesthesia, did not fit the group of patients with residual block due to the rapid recovery to values of $T_{4} / T_{1} \geq 0.9$. As for those who received rocuronium, they would probably still need a few minutes to reach full recovery.

The substitution of halogenated agent by a intravenous agent to maintain anesthesia until the children regain a safe value for the removal of the endotracheal tube had the intention to eliminate the interference of the inhalational anesthetic on the recovery time from neuromuscular block, and to keep the child stress free from the presence of the endotracheal tube ${ }^{16}$.

In awaken patients the use of submaximal current stimulation has been justified for being reliable and more comfortable. A study conducted in adult volunteers showed that stimulation with $20 \mathrm{~mA}$ scored 2 points on the Visual Analogue Scale of pain ${ }^{17}$. Although children were not awake, the application of lower current intensity stimulation was selected for being reliable, and because the lower intensity of pain caused would lead to little interference in the recovery from anesthesia.

The incidence of residual neuromuscular block with the different NDNMBs has well been described in the adult population in the recovery room after anesthesia. In children, however, data are scarce. In general, children present faster recovery of neuromuscular function after the use of NDNMB compared to adults ${ }^{18,19}$. This effect results from the lower power of NDNMBs, the faster spontaneous recovery and faster recovery following reversal of NDNMB observed in the pediatric patient ${ }^{20}$. Although studies have demonstrated that recovery from non-depolarizing neuromuscular blockade is faster in children than in adults ${ }^{9}$, anesthesiologists should be aware of residual block in pediatric patients.

Differently from the present study, Baxter et al. ${ }^{20}$ observed no residual block after use of atracurium, pancuronium and vecuronium in children, mostly between 2 and 10 years of age. The use of high dose of neostigmine for all children, the visual or tactile monitoring of neuromuscular blockade during surgery and the research of residual block in the recovery room, post anesthesia may have contributed to explain the different outcome. Also important is to highlight that the average time between the reversal of neuromuscular blockade and the record of $T_{4} / T_{1}$ by mechanomyography in the recovery room ranged from 15 to 18 minutes, and these authors do not mention the degree of neuromuscular blockade at the time of reversal of the blockade and the removal of the endotracheal tube.

Meretoja and Wirtavuori ${ }^{14}$ also studied the recovery of neuromuscular function after administration of atracurium or alcuronium in children in whose electromyography monitoring was hidden from the anesthesiologist 15 minutes before the end of anesthesia, so that he/she would continue making decisions based solely on clinical data. All children received $50 \mu \mathrm{g} \cdot \mathrm{kg}^{-1}$ of neostigmine for reversal of neuromuscular blockade. In the group receiving atracurium, the average time to recover $T_{4} / T_{1} \geq 0.9$ after reversal was 10 minutes, the average value of $\mathrm{T}_{4} / \mathrm{T}_{1}$ at the moment of removal of the endotracheal tube was $0.51 \pm 0.18$ and was less than $0.9(0.81 \pm 0.14)$ upon arrival in the recovery room after anesthesia ${ }^{10}$. In this study, the mean value of $\mathrm{T}_{1}$ at the time of reversal of the blockade was $81.6 \%$, corresponding to two or three responses to TOF, which was evaluated in our study.

Comparing these data with our study, the less time recorded for the recovery of $\mathrm{T}_{4} / \mathrm{T}_{1} \geq 0.9$ after injection of neostigmine can be explained by the fact that the reversal has been performed during higher degree of recovery from neuromuscular blockade. The incidence of patients with $T_{4} / T_{1}<0.9$ at extubation based on clinical criteria was also lower in this study. The option for early removal of the endotracheal tube based on clinical data could be attributed to the fact that the clinical criteria at that time had been determined solely by the patient's respiratory activity, and it is known that recovery of the diaphragmatic muscle occurs earlier than the recovery of hand muscles.

Monitoring neuromuscular function is important not only to avoid residual blockade and its consequences, but also because it prevents unnecessary reversal and their possible deleterious effects. In this study, the reversal of the blockade in patients who already had $T_{4} / T_{1} \geq 0.9$ was frequent, especially in the Rocuronium Group, although not statistically significant.

After reversal, the time to neuromuscular function recovery was the same for both groups. A considerable number of patients had the neuromuscular blockade incorrectly reversed when the blockade was still too deep or even already recovered.

At the moment of the decision to remove the endotracheal tube based on clinical criteria, $10 \%$ of children had $T_{4} / T_{1}<0.9$ regardless the blocker received. 